\title{
Aspirin promotes osteogenic differentiation of human dental pulp stem cells
}

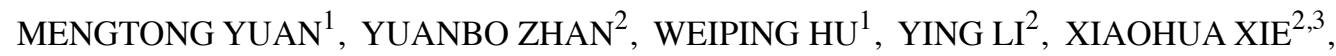 \\ NAN MIAO ${ }^{2}, \mathrm{HAN} \mathrm{JIN}^{2}$ and BIN ZHANG ${ }^{2,4}$ \\ ${ }^{1}$ Department of Prosthodontics, and ${ }^{2}$ Institute of Hard Tissue Development and Regeneration; \\ ${ }^{3}$ Department of Pediatric Dentistry, The Second Affiliated Hospital of Harbin Medical University, Harbin, \\ Heilongjiang 150086; ${ }^{4}$ Heilongjiang Academy of Medical Sciences, Harbin, Heilongjiang 150081, P.R. China
}

Received March 29, 2018; Accepted July 30, 2018

DOI: $10.3892 /$ ijmm.2018.3801

\begin{abstract}
Human dental pulp stem cells (hDPSCs) possess self-renewal and osteogenic differentiation properties, and have been used for orofacial bone regeneration and periodontal treatment. Aspirin has been demonstrated to enhance the regeneration of bone marrow mesenchymal stem cells (MSCs); however, the impact of aspirin on the osteogenic differentiation of hDPSCs remains unknown. In the present study, hDPSCs were characterized by flow cytometry, while their clonogenic potential and multipotency were assessed using alizarin red, Oil red $\mathrm{O}$ and alcian blue staining. The effect of aspirin on hDPSC viability was assessed using Cell Counting Kit-8 assay. Osteogenic capacity was examined by alkaline phosphatase activity, alizarin red staining, reverse transcription-polymerase chain reaction and western blotting. Furthermore, in vivo cranial defects were established in Sprague-Dawley rats to evaluate the effect of aspirin on hDPSC-based bone regeneration. Anorganic bovine bone was used as a bone replacement material and as the carrier for hDPSCs. New bone formation was observed through radiographic and histological analysis. The study demonstrated that hDPSCs expressed MSC markers and possessed multipotency in vitro. Aspirin was non-toxic to hDPSCs at a concentration of $\leq 100 \mu \mathrm{g} / \mathrm{ml}$ and enhanced the osteogenesis of hDPSCs in vitro. Aspirin significantly increased hDPSC-based bone formation in the rat cranial defect model at 8 or 12 weeks post-implantation $(\mathrm{P}<0.05)$. The data suggested that aspirin promotes the osteogenic potential of hDPSCs in vitro and in vivo. Overall, the present study indicated that aspirin improves the bone regeneration capacity of hDPSCs.
\end{abstract}

Correspondence to: Dr Bin Zhang, Institute of Hard Tissue Development and Regeneration, The Second Affiliated Hospital of Harbin Medical University, 246 Xue Fu Road, Harbin, Heilongjiang 150086, P.R. China

E-mail: zhangbinyzz@126.com

Key words: dental pulp stem cells, aspirin, osteogenic differentiation, regenerative medicine

\section{Introduction}

Human dental pulp stem cells (hDPSCs) are self-renewing, highly proliferative, multi-potent stem cells that are derived from enzymatically disaggregated adult human dental pulp (1). More than $90 \%$ of dental pulp cells (DPCs) display positive expression of the endothelial cell markers CD29, CD44 and CD146, while $>20 \%$ of DPCs express STRO-1, a mesenchymal stromal progenitor marker. Furthermore, DPCs have a negative expression of hematopoietic markers, including CD34, CD45 and CD133, and the endothelial marker, CD106 (2). Previous studies have demonstrated that hDPSCs exhibit a higher proliferative rate compared with human bone marrow stromal cells (hBMSCs), and are able to differentiate into odontoblasts/osteoblasts, chondrocytes, adipocytes and neural cells in vitro $(1,3,4)$. However, unlike BMSCs, DPSCs exhibit an odontogenic capability to form specific crystalline structures in mineralized nodules, similar to physiological dentin but distinct from bone structures (5). A previous study revealed that DPSCs, similar to mesenchymal stem cells (MSCs), possess immunomodulatory properties, and that Fas ligand governs the immunoregulatory properties of DPSCs in the induction of T-cell apoptosis (6). Taken together, hDPSCs may represent good sources of stem cells for experimental and clinical studies.

Aspirin, also known as acetylsalicylic acid (ASA), is the most widely used antipyretic, analgesic and non-steroidal anti-inflammatory drug (7). It affects multiple biological pathways by inhibiting cyclooxygenase and decreasing the production of prostaglandins (7). Previous studies have suggested that aspirin has the potential to promote bone regeneration (8-11). Aspirin increased the osteogenic capacity of bone marrow MSCs (BMMSCs) by targeting the telomerase activity, and inhibited osteoclast activity in mice (8). In addition, aspirin promoted BMMSC-based bone regeneration via inhibiting tumor necrosis factor- $\alpha$ and interferon- $\gamma$ production in skull defect models (9). Administration of aspirin was capable of improving BMSC-mediated calvarial bone regeneration in a porcine model (10), as well as osteogenic differentiation and immunomodulation mediated by stem cells derived from exfoliated deciduous teeth (11). Aspirin has also been demonstrated to enhance the function of periodontal ligament stem 
cells and may have regenerative dentistry applications (12). Combined use of aspirin and adipose-derived stem cells has been reported to partially reverse bone loss caused by castration in rats (13). In addition, regular administration of aspirin may have a moderate beneficial effect on bone mineral density in human patients (14). However, to the best of our knowledge, no previous studies have assessed the effect of aspirin on the osteogenic capacity of hDPSCs.

In the present study, the impact of aspirin on bone repair on hDPSC-seeded anorganic bovine bone (Bio-Oss), a tissue-engineered construct, was assessed in a rat calvarial defect model.

\section{Materials and methods}

Isolation and culture of hDPSCs. The present study was approved by the Ethical Board of the Second Affiliated Hospital of Harbin Medical University (Harbin, China). Written informed consent was obtained from the parents of the healthy donors (age, 14-18 years; 5 males and 2 females) undergoing orthodontic treatments. Briefly, cells were isolated from dental pulp tissue of extracted permanent teeth as previously described $(1,3,4)$. The cells were cultured in Human Mesenchymal Stem Cell Growth Medium (Cyagen Biosciences, Inc., Guangzhou, China) supplemented with $10 \%$ fetal bovine serum (FBS), $10 \mathrm{mM}$ glutamine and $100 \mathrm{U} / \mathrm{ml}$ penicillin-streptomycin at $37^{\circ} \mathrm{C}$ with $5 \% \mathrm{CO}_{2}$ in humidified incubator. At 3-4 days later, non-adherent cells were removed, and the medium was changed every 2 days thereafter. After $\sim 14$ days, colony formation unit-fibroblasts were formed (1), and various methodologies were performed.

A mixed colony culture was performed similarly to a multi-colony derived cell culture, as reported previously (15). Briefly, primary cells were passaged when colonies began to merge on days $12-14$ with $0.25 \%$ trypsin-EDTA (Gibco; Thermo Fisher Scientific, Inc., Waltham, MA, USA), following which all colonies and other cells were combined. For a picked colony culture, after 14-16 days, individual round colonies (>10 per flask) were selected and pooled together in a new flask. Upon reaching 80-90\% confluency, cells were collected with $0.25 \%$ trypsin-EDTA and passaged. At passage 3 (P3), adherent cells were collected, characterized and used in further experiments.

Flow cytometric analysis for cell characterization. P3 cells at confluence in $25-\mathrm{cm}^{2}$ flasks were collected and stained at $4^{\circ} \mathrm{C}$ for 30 min with antibodies against human CD29 (1:100; cat. no. 559882), CD44 (1:100; cat. no. 550989), CD133 (1:100; cat. no. 566593) and CD146 (1:100; cat. no. 550315; BD Biosciences, San Jose, CA, USA), as well as anti-STRO-1 antibody (1:50; cat. no. FAB1038G; R\&D Systems, Minneapolis, MN, USA). Subsequently, cells were analyzed using a FACS Calibur flow cytometer and Cell Quest software (BD Biosciences).

Multilineage differentiation of hDPSCs. P3 cells were seeded at $15 \times 10^{4}$ cells/well in 6-well plates (Corning Incorporated, Corning, NY, USA) and cultured in Human Mesenchymal Stem Cell Growth Medium (Cyagen Biosciences, Inc., Guangzhou, China) supplemented with $10 \%$ FBS, $10 \mathrm{mM}$ glutamine and $100 \mathrm{U} / \mathrm{ml}$ penicillin-streptomycin at $37^{\circ} \mathrm{C}$ with $5 \% \mathrm{CO}_{2}$ in a humidified incubator. Cells were then treated with osteogenic, chondrogenic and adipogenic induction medium for 2-3 weeks as previously reported (16). Trilineage differentiation of hDPSCs was evaluated by alizarin red, alcian blue and Oil Red O stains using a Human Mesenchymal Stem Cell Differentiation kit (Thermo Fisher Scientific, Inc.), in accordance with the manufacturer's protocol.

Aspirin cytotoxicity assay. The effect of aspirin on hDPSC viability was assessed using Cell Counting Kit-8 (Beyotime Institute of Biotechnology, Nantong, China), in accordance with the protocol provided by the manufacturer. Briefly, hDPSCs were seeded at a density of 4,000 cells/well in a 96-well flat-bottom plate (Corning Incorporated) in triplicate. Cells were maintained in $100 \mu \mathrm{l}$ standard medium containing $0,25,50,100,200$ or $400 \mu \mathrm{g} / \mathrm{ml}$ aspirin (Sigma-Aldrich; Merck KGaA, Darmstadt, Germany) for 24, 48 or $72 \mathrm{~h}$.

Alkaline phosphatase (ALP) staining and Alizarin red staining (ARS). Cells were cultured in Human Mesenchymal Stem Cell Osteogenic Differentiation Medium (Cyagen Biosciences, Inc.) containing $10 \% \mathrm{FBS}, 100 \mathrm{U} / \mathrm{ml}$ penicillin-streptomycin, $0.2 \mathrm{mM}$ ascorbate, $10 \mathrm{mM} \beta$-glycerophosphate and $10^{-7} \mathrm{M}$ dexamethasone. After $24 \mathrm{~h}$, aspirin $(0,25,50$ and $100 \mu \mathrm{g} / \mathrm{ml})$ was added to hDPSCs that were seeded at $15 \times 10^{4}$ cells/well in 6-well plates (Corning Incorporated).

ALP staining was conducted at day 14 using a BCIP/NBT staining kit (Beyotime Institute of Biotechnology, Shanghai, China), according to the manufacturer's protocol. NBT formazan was collected using $100 \mathrm{mM}$ cetylpyridinium chloride monohydrate (CPC; cat. no. C9002-25G; Sigma-Aldrich, Merck KGaA), and absorbance was read at $560 \mathrm{~nm}$ with a microplate reader (iMARK Microplate Absorbance Reader; Bio-Rad Laboratories, Inc., Hercules, CA, USA).

For the ARS assay, hDPSCs were cultured for 21 days and then fixed with $75 \%$ ethanol, followed by staining with $2 \%$ alizarin red (Beijing Solarbio Bioscience \& Technology Co., Ltd., Beijing, China; pH 4.2). Unbound and nonspecifically bound stain was removed by rinsing with distilled water. Calcium-bound stain was collected with $100 \mathrm{mM}$ $\mathrm{CPC}$, and absorbance was read at $560 \mathrm{~nm}$ with a microplate reader (iMARK Microplate Absorbance Reader; Bio-Rad Laboratories, Inc.).

Reverse transcription-quantitative polymerase chain reaction $(R T-q P C R)$. Cells were homogenized for RNA extraction using the RNeasy mini kit (Qiagen, Hilden, Germany). The RNA concentrations were measured by a Nanovue spectrophotometer (GE Healthcare Life Sciences, Marlborough, MA, USA), and the total RNA was then reverse-transcribed to cDNA using the Prime Script First Strand cDNA Synthesis kit (Takara Bio, Inc., Kusatsu, Japan) on the MxPro-Mx3000P Real-Time PCR System (Stratagene; Agilent Technologies, Inc., Santa Clara, CA, USA). PCR was then performed to determine the expression levels of target genes, and gene expression was normalized to that of $\beta$-actin. The relative differences in the PCR results were calculated by using the $2^{-\Delta \Delta \mathrm{Cq}}$ method (17). The thermocycling conditions were as follows: $95^{\circ} \mathrm{C}$ for $2 \mathrm{~min}$, followed by 
Table I. Primer sequences for quantitative polymerase chain reaction.

\begin{tabular}{lll}
\hline Gene & \multicolumn{1}{c}{ Reverse $\left(5^{\prime}-3^{\prime}\right)$} & Forward $\left(5^{\prime}-3^{\prime}\right)$ \\
\hline RUNX2 & CAGATGGGACTGTGGTTACTG & GAGGATTTGTGAAGACGGTTA \\
Col-I & AAGACGAAGACATCCCACCAA & CAGATCACGTCATCGCACAAC \\
OCN & AGGGCAGCGAGGTAGTGAAGA & AGAGGAGCAGAACTGGGGTTG \\
-actin & GGGCCGGACTCGTCATAC & CCTGGCACCCAGCACAAT
\end{tabular}

RUNX2, runt-related transcription factor 2; Col-I, collagen type I; OCN, osteocalcin.

40 cycles of $95^{\circ} \mathrm{C}$ for $15 \mathrm{sec}$ and $60^{\circ} \mathrm{C}$ for $30 \mathrm{sec}$. The primers used in this analysis are listed in Table I (Invitrogen; Thermo Fisher Scientific, Inc.).

Western blotting. The cells were harvested and proteins were extracted with RIPA lysis buffer (Beyotime, Shanghai, China). The protein concentration was determined using the BCA protein Assay (Beyotime, Shanghai, China). Equal aliquots of $40 \mu \mathrm{g}$ per sample were separated by sodium dodecyl sulfate-polyacrylamide gel electrophoresis (10-12\%) and transferred to polyvinylidene difluoride (PVDF) membranes (Millipore, Bedford, MA, USA). Following blocking in 5\% nonfat dry milk (dissolved in TBST, TBS plus $0.1 \%$ Tween-20) for $1 \mathrm{~h}$ at room temperature, the proteins of interest were probed with primary antibodies overnight at $4^{\circ} \mathrm{C}$ : osteocalcin (1:1,000; cat. no. ab13418; Abcam, Cambridge, UK), collagen I (1:1,000; cat. no. ab6308; Abcam), runt-related transcription factor 2 (RUNX2;1:1,000; cat. no. 12556; Cell Signaling Technology, Inc., Danvers, MA, USA) and $\beta$-actin $(1: 5,000$; cat. no. 3700; Cell Signaling Technology, Inc.). Subsequently, membranes were incubated with IRDye 800CW-labeled goat anti-rabbit IgG (H+L; 1:10,000; cat. no. 926-32211; LI-COR Biosciences, Lincoln, NE, USA) and goat anti-mouse IgG (1:10,000; H+L; cat. no. 926-32210; LI-COR Biosciences) for $1 \mathrm{~h}$ at room temperature. The blots were then visualized using an Infrared Imaging System (LI-COR Biosciences, Lincoln, NE, USA). The band density was quantified using Odyssey software version 3.0 (LI-COR Biosciences) and normalized to $\beta$-actin.

Generation of rat calvarial bone defects and transplantation. The animal studies were approved by the Ethical Board of the Second Affiliated Hospital of Harbin Medical University. The 40 adult male Sprague-Dawley rats aged 9-10 weeks (200-250 g) used in this study were supplied by the Animal Center of the Second Affiliated Hospital of Harbin Medical University (Harbin, China; No. SYXK, 2013-002). The rats were housed in an animal facility with $20-23^{\circ} \mathrm{C}, 40-60 \%$ humidity and a 12-h light/dark cycle. Standard laboratory chow and water ad libitum were supplied. hDPSCs were cultured in Human Mesenchymal Stem Cell Osteogenic Differentiation Medium prior to implantation into the cranial defects. Briefly, Sprague-Dawley rats were anesthetized with intraperitoneal injection of $300 \mathrm{mg} / \mathrm{kg}$ chloral hydrate (10\%; cat. no. C8383; Sigma-Aldrich; Merck KGaA), and then bicortical defects of 5-mm diameter were created with a stainless-steel trephine (18).
The rats were randomly assigned to four different groups $(n=10$ per group) with the following: i) Untreated group, unfilled defects; ii) BO group, 0.02 g Bio-Oss (Geistlich Pharma AG, Wolhusen, Switzerland) only; iii) DPSC/BO group, hDPSCs (8x10 $10^{6}$ cells) + Bio-Oss; and iv) DPSC/BO/ASA group, hDPSCs (8x10 $0^{6}$ cells) treated with $100 \mu \mathrm{g} / \mathrm{ml}$ aspirin for 3 days using Bio-Oss as a carrier. Following the placement of the materials, the surgical site was covered with a native collagen membrane (Biogide ${ }^{\circledR}$; Geistlich Pharma AG), and the soft tissues were closed with sutures. All the animals received a single dose of ampicillin (100 mg/kg; A6920, Beijing Solarbio Bioscience \& Technology Co., Ltd.) $12 \mathrm{~h}$ post-surgery. The rats were sacrificed with an overdose of pentobarbital sodium $(100 \mathrm{mg} / \mathrm{kg}$; intravenous) at 8 or 12 weeks postoperatively, and the calvaria were immediately excised and fixed in $4 \%$ neutral-buffered formaldehyde.

Electron microscopy. To observe cells adhesion on scaffolds in vitro, hDPSCs were seeded on Bio-Oss at a density of $8 \times 10^{6}$ cells per $0.02 \mathrm{~g}$ Bio-Oss and cultured for $24 \mathrm{~h}$. The samples were fixed in $2.5 \%$ glutaraldehyde and evaluated by scanning electron microscopy (SEM; S-3400; Hitachi, Ltd., Tokyo, Japan).

Radiography and micro-computed tomography (CT) scanning. Explanted calvaria samples were radiographed by X-ray (Faxitron Bioptics LLC, Tucson, AZ, USA) and scanned by a micro-CT scanner ( $\mu$ CT35; Scanco Medical AG, Bassersdorf, Switzerland) to examine the new bone within the defect region.

Histology. The specimens were fixed, decalcified and paraffin-embedded. Sections $(4-\mu \mathrm{m})$ were prepared and then stained with hematoxylin and eosin (H\&E) or with Masson's trichrome (MTS). New bone formation within the defect was measured histomorphometrically using an image analysis software (Image Pro Plus, version 7.0; Media Cybernetics, Inc., Bethesda, MD, USA).

Statistical analysis. The results are reported as the mean \pm standard deviation of three independent experiments. The data were analyzed using GraphPad Prism software, version 6.0 (GraphPad Software, Inc., La Jolla, CA, USA). Multiple comparisons were performed by one-way analysis of variance followed by Tukey's test. $\mathrm{P}<0.05$ was considered to indicate a statistically significant difference. 

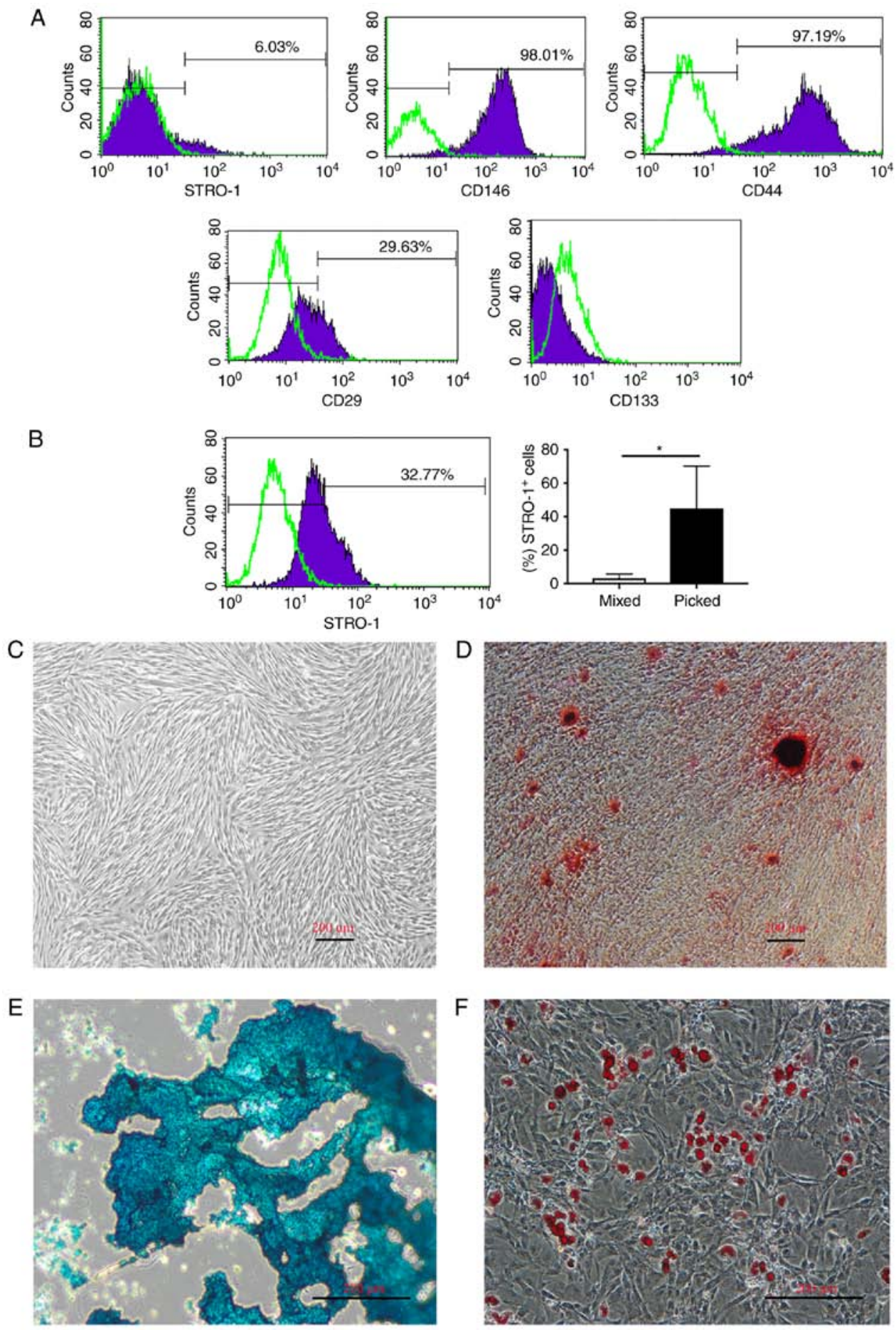

Figure 1. Characterization of hDPSCs. (A) Flow cytometric analysis of cultured hDPSCs at P3 revealed the expression of STRO-1 (6.03\%), CD146 (98.01\%), CD44 (97.19\%) and CD29 (29.63\%). CD133 expression was negative. (B) Primary hDPSCs were cultured using two methods, namely mixed colonies and picked colonies. The later method strongly raised the percentage of STRO-1 ${ }^{+}$cells (32.77\%) at P3 (44.9 vs. 3.25\%). (C) Morphology is typical of fibroblast-like cell (scale bar, $200 \mu \mathrm{m}$ ). Multilineage differentiation of hDPSCs was examined using (D) osteogenic (alizarin red) staining, (E) chondrogenic (alcian blue) staining, and (F) adipogenic (Oil red O) staining (scale bar, $200 \mu \mathrm{m}$ ). "P<0.05. hDPSCs, human dental pulp stem cells; P3, passage 3.

\section{Results}

hDPSCs express MSC markers and have multilineage differentiation potential in vitro. hDPSCs were characterized at P3 by flow cytometry, and the majority of cells were found to express STRO-1, CD146, CD44 and CD29. By contrast, CD133 expression was not evident (Fig. 1A). Furthermore, hDPSCs were cultured by picking established colonies at passage 0 , which increased the percentage of STRO- $1^{+}$cells at P3 compared with the mixed colony culture method (44.9 vs. $3.25 \%$; $\mathrm{P}<0.05)$. STRO-1 was expressed by $\leq 70 \%$ of cells (mean value, 44.9\%; Fig. 1B). The cells proliferated rapidly following the subculture and homogeneously exhibited a fibroblast-like spindle shape (Fig. 1C). Cells were positive for ARS, alcian blue staining and Oil red $\mathrm{O}$ staining in response to osteogenic, chondrogenic and adipogenic induction, respec- 
A

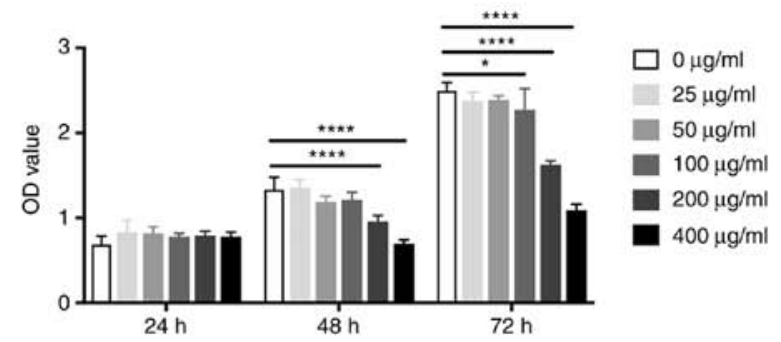

B
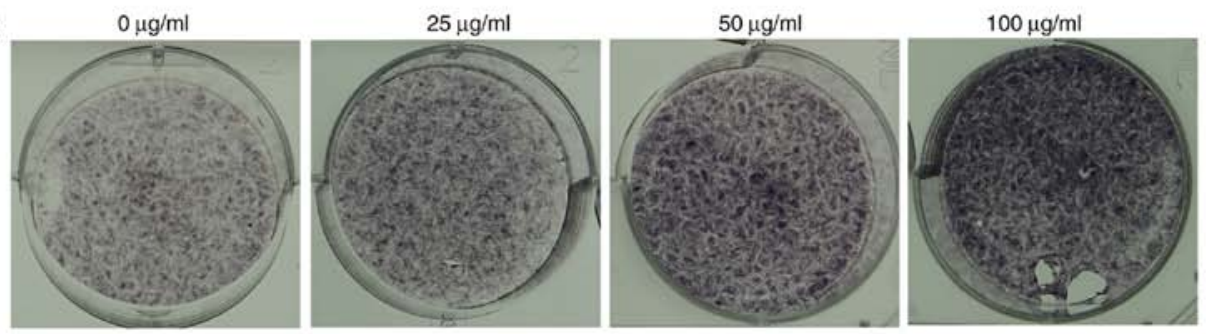

C
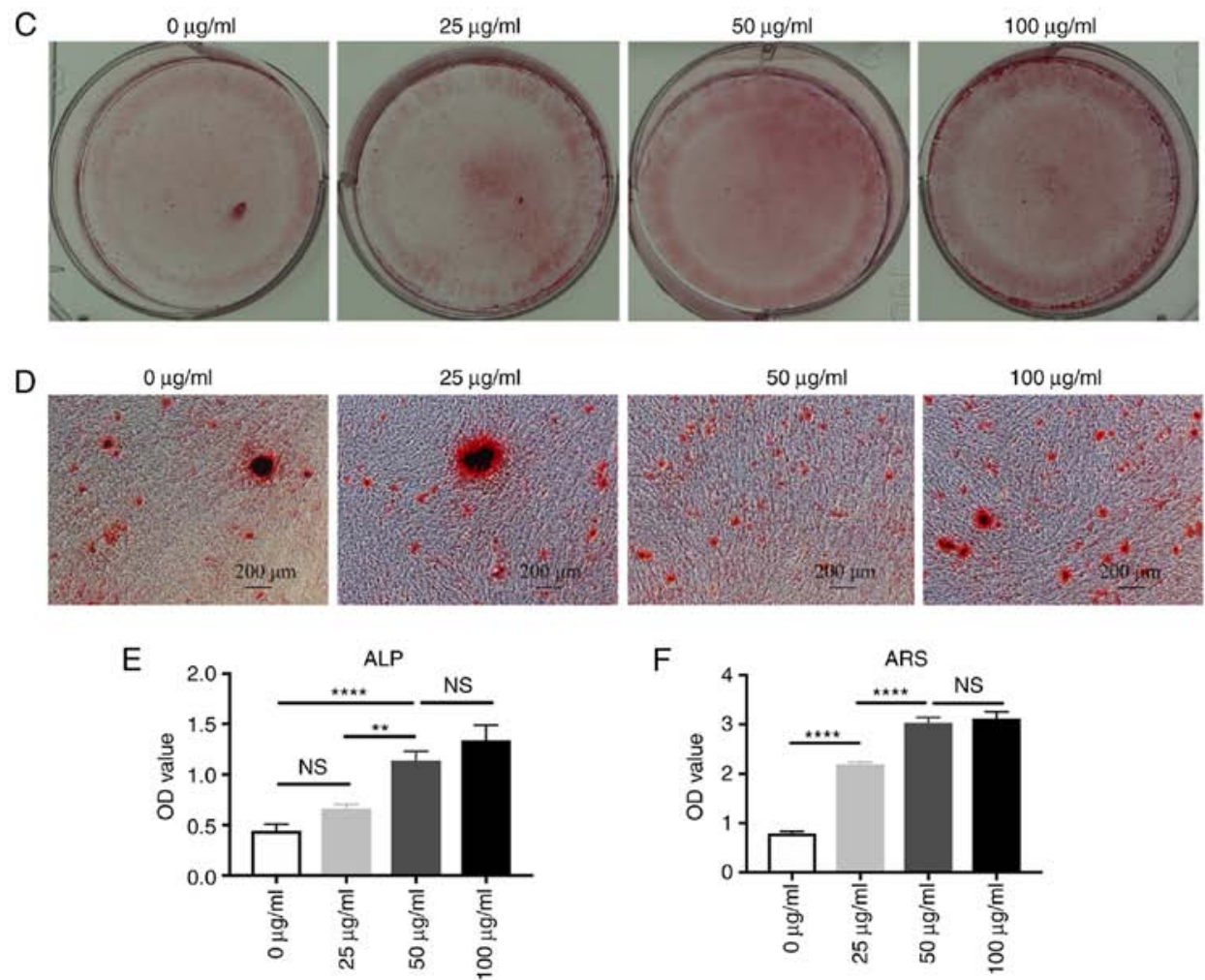

Figure 2. Aspirin increases the mineralization of hDPSCs in vitro. (A) Aspirin was non-toxic to hDPSCs at a concentration of $\leq 100 \mu \mathrm{g} / \mathrm{ml}$, as shown by CCK-8 assay. (B) hDPSCs treated with different doses of aspirin $(0,25,50$ and $100 \mu \mathrm{g} / \mathrm{ml})$, exhibiting increased ALP activity at 14 days and (C) capability of forming mineralized nodules at 21 days in a dose-dependent manner. (D) A magnified view of the mineralized matrix by alizarin red staining. Scale bar, $200 \mu$ m. Significantly increased (E) ALP activity and (F) calcium deposition were observed in cells treated with $100 \mu \mathrm{g} / \mathrm{ml}$ aspirin, compared with the untreated group. ${ }^{*} \mathrm{P}<0.05,{ }^{* *} \mathrm{P}<0.01$ and ${ }^{* * * *} \mathrm{P}<0.0001$. hDPSCs, human dental pulp stem cells; CCK-8, Cell Counting Kit-8; ALP, alkaline phosphatase; OD, optical density; ns, non-significant.

tively (Fig. 1D-F). Taken together, these results indicated that hDPSCs possess MSC properties, and that the picked colony culture method is suitable for multipotent hDPSC culture and augments the STRO-1 ${ }^{+}$subpopulation.

Aspirin enhances osteogenic differentiation of hDPSCs in vitro. The present study first assessed the toxicity of aspirin on hDPSCs in vitro. Aspirin at concentrations of $\leq 100 \mu \mathrm{g} / \mathrm{ml}$ had no significant effect on the viability of hDPSCs within $72 \mathrm{~h}$, while 200 and $400 \mu \mathrm{g} / \mathrm{ml}$ aspirin caused a significant decrease in cell viability at 48 and $72 \mathrm{~h}$ (Fig. 2A). Therefore, $\leq 100 \mu \mathrm{g} / \mathrm{ml}$ aspirin was used to treat hDPSCs in the following experiments. Next, it was demonstrated that aspirin increased ALP activity, whose expression by functional osteoblasts precedes mineralization (19), in a dose-dependent manner (Fig. 2B and E). The hDPSC-osteoinductive function of aspirin was then assessed by ARS, and the results revealed that 21-day aspirin treatment at doses of 50 and $100 \mu \mathrm{g} / \mathrm{ml}$ significantly augmented mineralized nodule formation by $\sim 2-3$ fold (Fig. 2C, D and F).

The study further examined the effects of aspirin on the expression levels of osteogenesis-associated markers, including RUNX2, collagen I and osteocalcin, in 

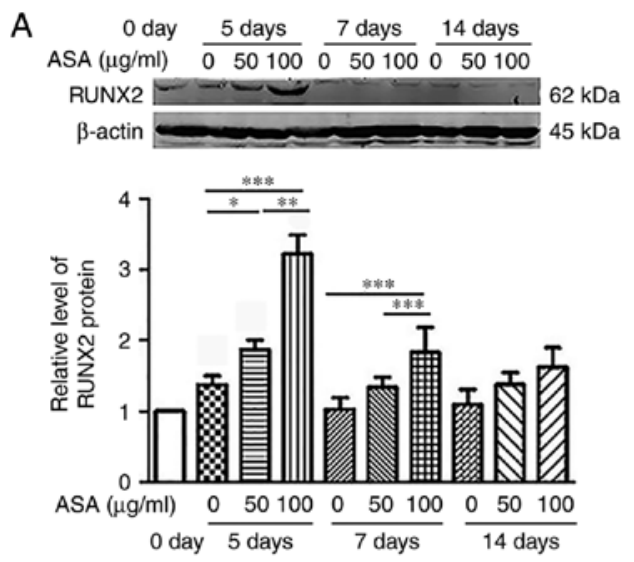
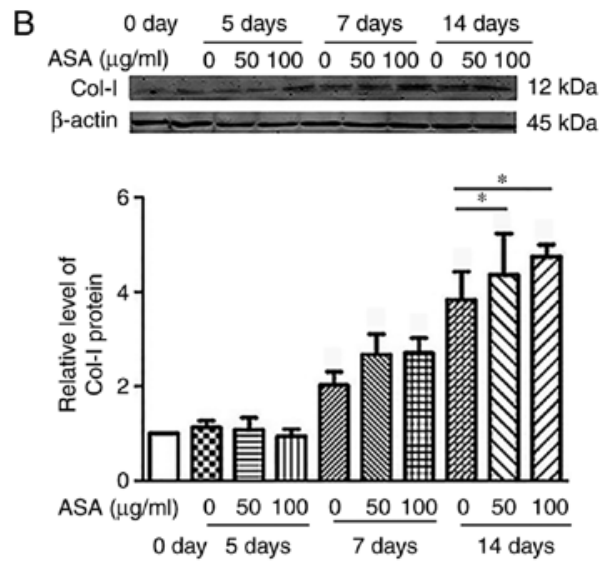
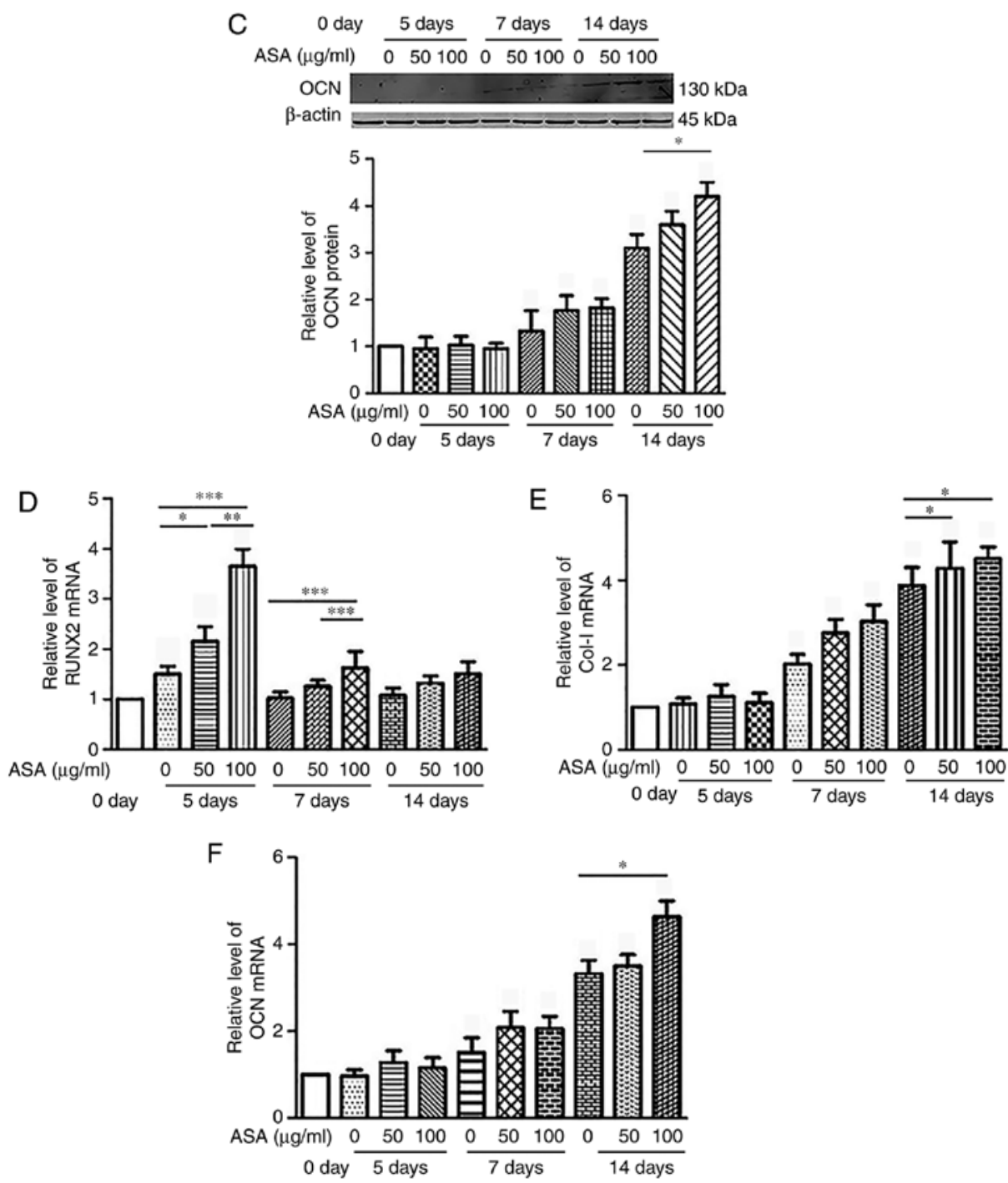

Figure 3. Aspirin (ASA) enhances the expression levels of osteogenic markers of hDPSCs in vitro. (A) RUNX2, (B) Col-I and (C) OCN protein levels were examined by western blotting, while (D) RUNX2, (E) Col-I and (F) OCN mRNA levels were examined by reverse transcription-polymerase chain reaction. These levels were elevated in the aspirin treatment groups compared with the untreated group on the same day. Statistical analysis was performed using one-way analysis of variance. Data were presented as the mean \pm standard deviation. ${ }^{*} \mathrm{P}<0.05,{ }^{* *} \mathrm{P}<0.01$ and ${ }^{* * * *} \mathrm{P}<0.001$. hDPSCs, human dental pulp stem cells; RUNX2, runt-related transcription factor 2; Col-I, collagen type I; OCN, osteocalcin.

hDPSCs (Fig. 3). The results demonstrated that high doses of ASA $(100 \mu \mathrm{g} / \mathrm{ml})$ significantly improved the osteogenic differentiation of hDPSCs compared with the untreated groups in vitro. RUNX2 upregulation was observed on days 5 and 7 (Fig. 3A and D), while collagen I (Fig. 3B and E) and osteocalcin (Fig. 3C and F) were significantly upregulated on day 14, at the protein and mRNA levels (Fig. 3). Cumulatively, these data suggested that aspirin enhanced the osteogenic differentiation potential of hDPSCs in the ex vivo culture. 

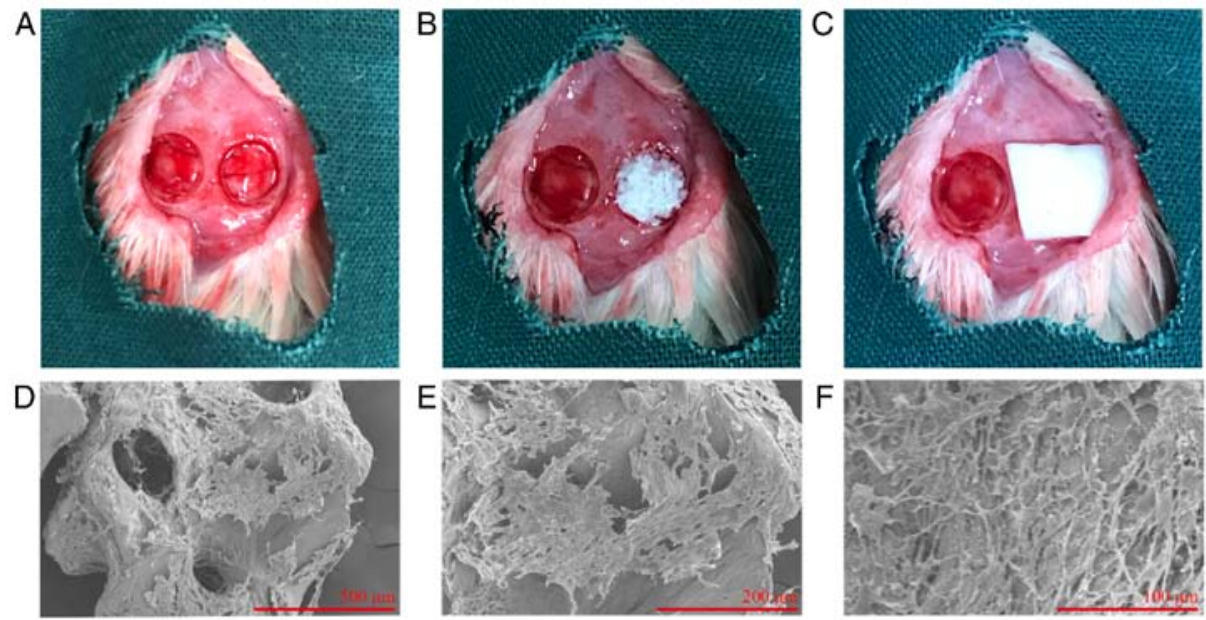

Figure 4. Rat calvarial bone defects and microscopic analysis. (A-C) Generation of rat calvarial bone defects and transplantation. (D-F) Microscopic analysis of hDPSCs cultured on Bio-Oss scaffolds was performed. hDPSCs dispersed as a monolayer and covered parts of the Bio-Oss surface, evaluated by scanning electron microscopy. (D) magnification, x100 (scale bar, $500 \mu \mathrm{m}$ ), (E) magnification, x200 (scale bar, $200 \mu \mathrm{m}$ ) and (F) magnification, x500 (scale bar, $100 \mu \mathrm{m}$ ). hDPSCs, human dental pulp stem cells.

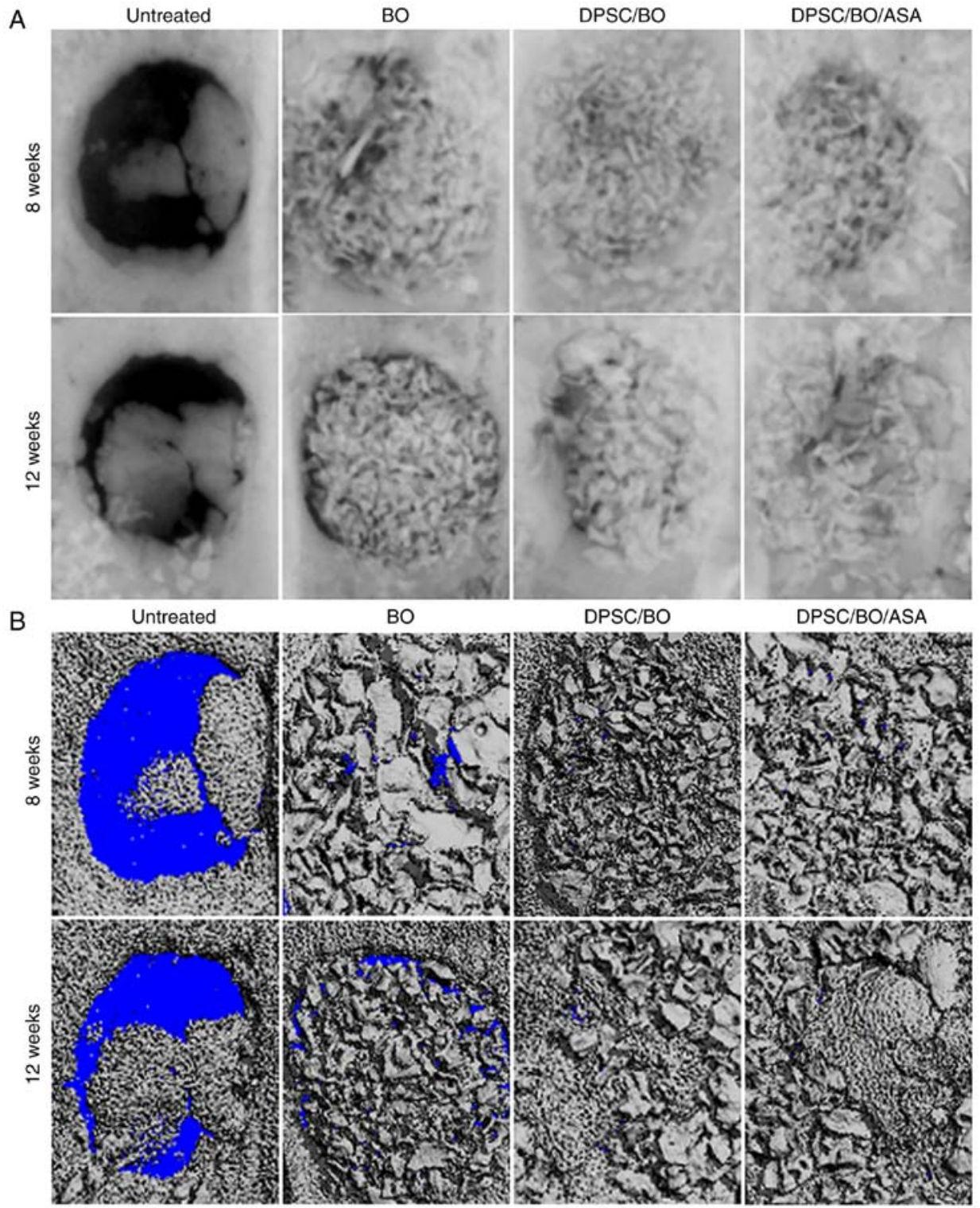

Figure 5. Aspirin promotes hDPSC-based calvarial bone regeneration in rats, as demonstrated by radiographs. (A) X-ray and (B) micro-computed tomography images revealed incomplete healing after 8 or 12 weeks in untreated rats. Bio-Oss significantly supported bone healing in the cranial defect model, while hDPSCs seeded on Bio-Oss further augmented the high-density area. hDPSC, human dental pulp stem cell; BO, Bio-Oss; ASA, acetylsalicylic acid (aspirin). 
A

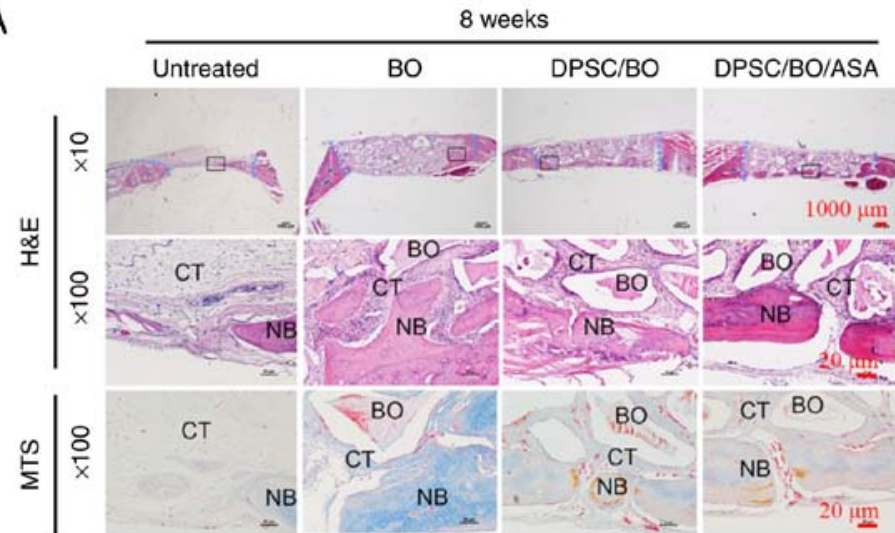

C

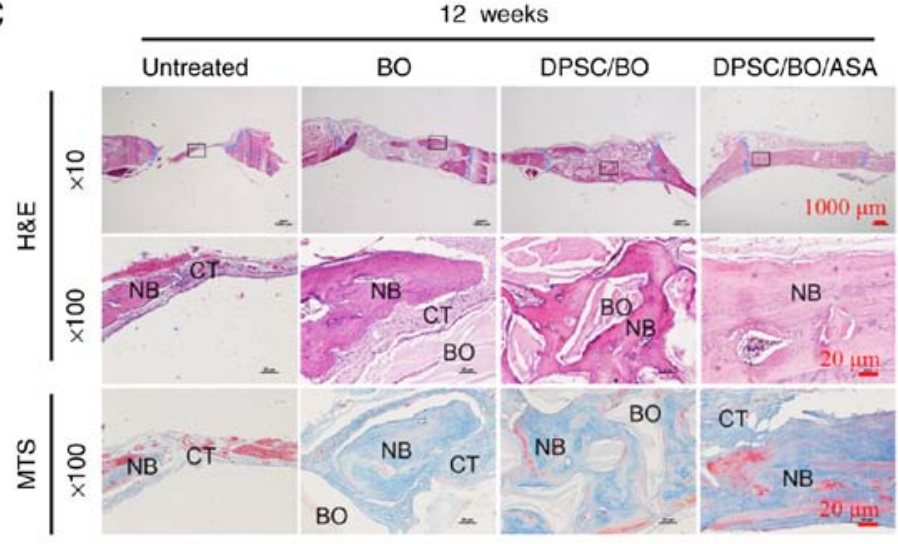

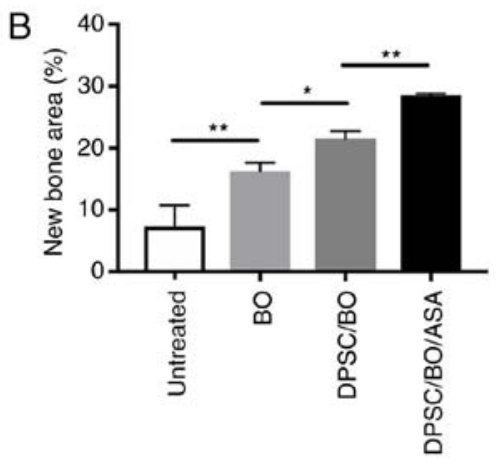

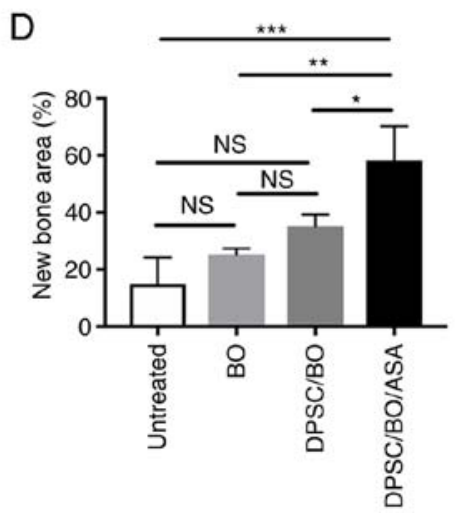

Figure 6. Aspirin treatment significantly improves hDPSC-based bone formation in a rat calvavial defect model. (A) Calvarial bone specimens that were untreated, or treated with BO, hDPSCs+BO or hDPSCs+BO+ASA were retrieved at 8 weeks post-surgery, and (B) quantitative analyses, based on H\&E staining, of the percentage of new bone formation between different groups was performed. (C) Calvarial bone specimens collected at 12 weeks post-surgery in the different groups, and (D) H\&E-based quantitative analysis of new bone formation. Sections were stained with H\&E and MTS, and images were captured at low (x10; scale bar, 1,000 $\mu \mathrm{m}$ ) and high magnification (x100; scale bar, $20 \mu \mathrm{m}$ ). The edge of the defects was shown in blue in the low magnification images. $\mathrm{H} \& \mathrm{E}$ staining results were analyzed using Image-Pro Plus 6.0 software. ${ }^{*} \mathrm{P}<0.05,{ }^{* *} \mathrm{P}<0.01$ and ${ }^{* * *} \mathrm{P}<0.001$. hDPSC, human dental pulp stem cell; BO, Bio-Oss; ASA, acetylsalicylic acid (aspirin); NB, new bone; CT, connective tissue; H\&E, hematoxylin and eosin; MTS, Masson's trichrome.

Aspirin improves hDPSC-based bone formation in vivo. Using a rat calvarial defect model (Fig. 4A-C), it was confirmed that aspirin enhanced the bone-forming capacity of hDPSCs in vivo. hDPSCs were seeded on Bio-Oss and incubated for $24 \mathrm{~h}$ prior to SEM examination. It was observed that hDPSCs dispersed as a monolayer and covered parts of the Bio-Oss surface (Fig. 4D-F). In addition, radiographs revealed incomplete healing after 8 or 12 weeks in untreated rats (Fig. 5A and B). By contrast, Bio-Oss significantly increased bone healing in the cranial defect model, and hDPSCs seeded on Bio-Oss further augmented the high-density area.

To assess whether aspirin improved new bone formation, the calvarial bone specimens were histologically analyzed, and aspirin was found to enhance the hDPSC-mediated bone formation in vivo (Fig. 6A-D). H\&E and MTS staining revealed minimal new bone formation around the margins of the native bone in the untreated groups, where soft fibrous tissue filled the center of the defect area. Limited new bone formation was evident in the BO group, while a moderate amount of bone formation was noted in defects treated with DPSC/BO. Aspirin treatment resulted in abundant mineralized tissue formation at 8 and 12 weeks post-surgery (Fig. 6A and C). According to the H\&E staining, more new bone was evident at the bottom of the defect during the earlier period of bone formation, and the untreated group exhibited significantly less new bone density in comparison with the other three groups at 8 weeks. Aspirin-treated hDPSCs exhibited significantly increased new bone formation, with woven/lamellar features in the defect area, as compared with the other groups at 12 weeks (Fig. 6B and D), indicating a pro-osteogenic effect exerted by aspirin $(\mathrm{P}<0.05)$. These findings suggested that aspirin improved bone formation when hDPSCs were seeded Bio-Oss in a rat cranial defect model.

\section{Discussion}

hDPSCs are easily isolated from the teeth of healthy donors undergoing orthodontic treatments. In addition, hDPSCs proliferate faster than hBMSCs and can differentiate into multiple cell types, particularly osteogenic cells (20). Previous studies have demonstrated that hDPSCs possess the ability to differentiate into neural, odontogenic and osteogenic cells, with the latter two cell types being able to form mineralized nodules in vitro $(21,22)$. It has also been demonstrated that multi-colony-derived DPSCs have a potential capacity to proliferate in vitro and to regenerate dentin in vivo, as compared with single-colony-derived strains (3). In the present study, hDPSCs were cultured using mixed colony culture and 
picked colony culture methods at passage 0 , the two of which initiated successful cell expansion.

DPSCs have been reported to express CD10, CD29, CD44, CD59, CD73, CD90, CD105, CD150 and CD166, but not hematopoietic cell surface markers, such as CD45, CD14, CD18, CD24, CD34 or CD133 (6,23). A previous study revealed that STRO-1 and CD146 (also known as melanoma cell adhesion molecule or MUC18) function as markers for pre-osteogenic stem cells and high-purity BMMSCs, respectively $(24,25)$. The STRO $-1^{+}$fraction represented $\sim 6 \%$ of the total pulp cells, which have higher capacity for colony formation and osteoblast differentiation $(24,26)$. In the present study, it was demonstrated that STRO-1 was expressed by $\leq 70 \%$ of hDPSCs (mean value, $44.9 \%$ ). This finding is consistent with previous studies reporting that STRO-1 is a marker of pre-osteogenic populations, the expression of which is lost upon cell proliferation and differentiation into mature osteoblasts.

Although DPSCs have similar characteristics to BMMSCs, DPSCs exhibit reduced osteogenic and adipogenic potentials compared with BMMSCs $(1,6)$. The current study focused on investigating various potent regulators, such as cytokines or drugs, to regulate the differentiation of DPSCs. It has been reported that aspirin has an anti-proliferative effect on BMMSCs at high concentrations, but not at low ones (50-200 $\mu \mathrm{g} / \mathrm{ml})(27)$. This is consistent with the results of the present study, which revealed that aspirin exerted little effect on the number of hDPSCs at low concentrations $(<100 \mu \mathrm{g} / \mathrm{ml})$. That is likely due to different resources and protocols used for MSC culture that may result in different responses to aspirin.

DPSCs and BMSCs exhibited a similar expression pattern of bone markers, including ALP, collagen I, osteocalcin and osteopontin (1). RUNX2 is the early osteoblastic transcription factor (28). Collagen I, the dominant fibrous protein in hard tissues, such as bone and dentin, is secreted by mature osteoblasts. Osteoblasts produce a collagen extracellular matrix that becomes mineralized through various signaling molecules, particularly bone sialoprotein, osteopontin and osteocalcin (29). Osteocalcin is considered to be the latest-functioning expression marker in mature osteoblasts (28). It has been demonstrated that aspirin can affect the expression levels of those markers. For instance, Liu et al (11) reported that low doses of aspirin (10 and $50 \mu \mathrm{g} / \mathrm{ml})$ treatment, but not a high dose $(200 \mu \mathrm{g} / \mathrm{ml})$, upregulated RUNX2 and ALP expression levels. This was consistent with the results of the present study, which demonstrated that the expression levels of RUNX2, collagen I and osteocalcin were elevated when the cells were treated with aspirin. This suggests that aspirin enhanced the osteogenic capacity of hDPSCs in vitro.

Bone engineering in craniomaxillofacial surgery requires scaffold/supporting materials, adequate target cells and osteogenesis-inducing factors (30). Several in vivo experiments have indicated that DPSCs have potential applications in bone engineering (31), while aspirin promotes the bone-forming ability of BMSCs (9) and stem cells from human exfoliated deciduous teeth (11). The present study used Bio-Oss as a carrier to transplant hDPSCs into rat cranial defects with or without aspirin treatment. It was identified that aspirin treatment promoted hDPSC-induced bone regeneration. Bio-Oss is a commercially available bone substitute with osteoconductive properties that supports new bone formation for implant dentistry and maxil- lofacial surgery $(32,33)$. However, a consensus regarding the biodegradation of Bio-Oss is yet to be reached. A number of investigators have observed that the bovine bone mineral is replaced by the newly formed bone $(34,35)$, while others have indicated that the resorption process of Bio-Oss was markedly slow $(36,37)$. Histological and histomorphometrical analyses in the current study indicated that the majority of the Bio-Oss particles were surrounded by new bone formation, indicating that Bio-Oss was biocompatible and osteoconductive. In addition, according to the $\mathrm{H} \& \mathrm{E}$ staining results, new bone was located adjacent to the dura mater during the early period of bone formation. Similarly, it has previously been reported that new bone formation localized to the area of the scaffold adjacent to the dura mater potentially indicates strong paracrine signaling between the underlying dura mater and the cells within the defect (38).

In conclusion, the present study demonstrated that hDPSCs exhibit stem cell properties, including expression of MSC markers and a capacity to differentiate into multiple cell lineages in vitro. This suggests that hDPSCs may be a feasible source of MSCs. Furthermore, aspirin improved the potential of osteogenic differentiation of hDPSCs in vitro and in vivo. Thus, the present study provides a promising basis for an hDPSC-based bone regeneration therapy.

\section{Acknowledgements}

The authors would like to thank Dr Quan Liu (Harbin Medical University, Harbin, China for the critical revision of this article.

\section{Funding}

This study was supported by the National Natural Science FoundationofChina(grantno.81570951 and81500816),theSpecial Foundation for Sino-Russian Translational Medicine Research Center of Harbin Medical University (grant no. CR201412 and CR201504), the Natural Science Foundation of Heilongjiang Province of China (grant no. H2015103), the Research Innovation Fund of Harbin Medical University (grant no. 2016LCZX1 9), the Science Foundation of the Second Affiliated Hospital of Harbin Medical University (grant no. CX2016-20), and the Research Grant of Health and Family Planning Commission of Heilongjiang Province (grant no. 2016-060).

\section{Availability of data and materials}

All data generated or analyzed during this study are included in the published article.

\section{Authors' contributions}

BZ and YL conceived and designed the experiments. MY, YZ, $\mathrm{NM}$ and $\mathrm{XX}$ performed the experiments. MY, WH and HJ analyzed the data. MY wrote the paper.

\section{Ethics approval and consent to participate}

The experimental protocols of the present study were approved by the Ethical Board of the Second Affiliated Hospital of Harbin Medical University (Harbin, China). 


\section{Patient consent for publication}

Not applicable.

\section{Competing interests}

The authors declare that they have no conflicts of interest.

\section{References}

1. Gronthos S, Mankani M, Brahim J, Robey PG and Shi S: Postnatal human dental pulp stem cells (DPSCs) in vitro and in vivo. Proc Natl Acad Sci USA 97: 13625-13630, 2000.

2. Wei X, Ling J, Wu L, Liu L and Xiao Y: Expression of mineralization markers in dental pulp cells. J Endod 33: 703-708, 2007.

3. Gronthos S, Brahim J, Li W, Fisher LW, Cherman N, Boyde A DenBesten P, Robey PG and Shi S: Stem cell properties of human dental pulp stem cells. J Dent Res 81: 531-535, 2002.

4. Liu H, Gronthos S and Shi S: Dental pulp stem cells. Methods Enzymol 419: 99-113, 2006.

5. About I, Bottero MJ, de Denato P, Camps J, Franquin JC and Mitsiadis TA: Human dentin production in vitro. Exp Cell Res 258: 33-41, 2000.

6. Zhao Y, Wang L, Jin Y and Shi S: Fas ligand regulates the immunomodulatory properties of dental pulp stem cells. J Dent Res 91: 948-954, 2012

7. Smith JB and Willis AL: Aspirin selectively inhibits prostaglandin production in human platelets. Nat New Biol 231: 235-237, 1971

8. Yamaza T, Miura Y, Bi Y, Liu Y, Akiyama K, Sonoyama W, Patel V, Gutkind S, Young M, Gronthos S, et al: Pharmacologic stem cell based intervention as a new approach to osteoporosis treatment in rodents. PLoS One 3: e2615, 2008.

9. Liu Y, Wang L, Kikuiri T, Akiyama K, Chen C, Xu X, Yang R, Chen W, Wang S and Shi S: Mesenchymal stem cell-based tissue regeneration is governed by recipient $\mathrm{T}$ lymphocytes via IFN- $\gamma$ and TNF- $\alpha$. Nat Med 17: 1594-1601, 2011.

10. Cao Y, Xiong J, Mei S, Wang F, Zhao Z, Wang S and Liu Y: Aspirin promotes bone marrow mesenchymal stem cell-based calvarial bone regeneration in mini swine. Stem Cell Res Ther 6: 210, 2015.

11. Liu Y, Chen C, Liu S, Liu D, Xu X, Chen X and Shi S: Acetylsalicylic acid treatment improves differentiation and immunomodulation of SHED. J Dent Res 94: 209-218, 2015.

12. Abd Rahman F, Mohd Ali J, Abdullah M, Abu Kasim NH and Musa S: Aspirin enhances osteogenic potential of periodontal ligament stem cells (PDLSCs) and modulates the expression profile of growth factor-associated genes in PDLSCs. J Periodontol 87: 837-847, 2016.

13. Liu H, Li W, Liu Y, Zhang X and Zhou Y: Co-administration of aspirin and allogeneic adipose-derived stromal cells attenuates bone loss in ovariectomized rats through the anti-inflammatory and chemotactic abilities of aspirin. Stem Cell Res Ther 6: 200, 2015.

14. Bauer DC, Orwoll ES, Fox KM, Vogt TM, Lane NE, Hochberg MC, Stone K and Nevitt MC: Aspirin and NSAID use in older women: Effect on bone mineral density and fracture risk. J Bone Miner Res 11: 29-35, 1996.

15. Kuznetsov SA, Krebsbach PH, Satomura K, Kerr J, Riminucci M, Benayahu D and Robey PG: Single-colony derived strains of human marrow stromal fibroblasts form bone after transplantation in vivo. J Bone Miner Res 12: 1335-1347, 1997.

16. Lei M, Li K, Li B, Gao LN, Chen FM and Jin Y: Mesenchymal stem cell characteristics of dental pulp and periodontal ligament stem cells after in vivo transplantation. Biomaterials 35: 6332-6343, 2014

17. Livak KJ and Schmittgen TD: Analysis of relative gene expression data using real-time quantitative PCR and the $2^{-\Delta \Delta C}$ method. Methods 25: 402-408, 2001.

18. Spicer PP, Kretlow JD, Young S, Jansen JA, Kasper FK and Mikos AG: Evaluation of bone regeneration using the rat critical size calvarial defect. Nat Protoc 7: 1918-1929, 2012.
19. Shi S, Gronthos S, Chen S, Reddi A, Counter CM, Robey PG and Wang CY: Bone formation by human postnatal bone marrow stromal stem cells is enhanced by telomerase expression. Nat Biotechnol 20: 587-591, 2002.

20. Nuti N, Corallo C, Chan BM, Ferrari M and Gerami-Naini B: Multipotent differentiation of human dental pulp stem cells: A literature review. Stem Cell Rev 12: 511-523, 2016.

21. Liu M, Sun Y, Liu Y, Yuan M, Zhang Z and Hu W: Modulation of the differentiation of dental pulp stem cells by different concentrations of $\beta$-glycerophosphate. Molecules 17: 1219-1232, 2012.

22. Wang Y, Yao J, Yuan M, Zhang Z and $\mathrm{Hu}$ W: Osteoblasts can induce dental pulp stem cells to undergo osteogenic differentiation. Cytotechnology 65: 223-231, 2013.

23. Ferro F, Spelat R, Beltrami AP, Cesselli D and Curcio F: Isolation and characterization of human dental pulp derived stem cells by using media containing low human serum percentage as clinical grade substitutes for bovine serum. PLoS One 7: e48945, 2012.

24. Shi S and Gronthos S: Perivascular niche of postnatal mesenchymal stem cells in human bone marrow and dental pulp. J Bone Miner Res 18: 696-704, 2003.

25. Gronthos S and Zannettino AC: A method to isolate and purify human bone marrow stromal stem cells. Methods Mol Biol 449: 45-57, 2008.

26. Yu J, He H, Tang C, Zhang G, Li Y, Wang R, Shi J and Jin Y: Differentiation potential of STRO- $1^{+}$dental pulp stem cells changes during cell passaging. BMC Cell Biol 11: 32, 2010.

27. Tang J, Xiong J, Wu T, Tang Z, Ding G, Zhang C, Wang S and Liu Y: Aspirin treatment improved mesenchymal stem cell immunomodulatory properties via the $15 \mathrm{~d}-\mathrm{PGJ}_{2} / \mathrm{PPAR} \gamma / \mathrm{TGF}-\beta 1$ pathway. Stem Cells Dev 23: 2093-2103, 2014.

28. Sun L, Blair HC, Peng Y, Zaidi N, Adebanjo OA, Wu XB, Wu XY, Iqbal J, Epstein S, Abe E, et al: Calcineurin regulates bone formation by the osteoblast. Proc Natl Acad Sci USA 102 17130-17135, 2005.

29. Wiesmann HP, Meyer U, Plate U and Höhling HJ: Aspects of collagen mineralization in hard tissue formation. Int Rev Cytol 242: 121-156, 2005.

30. Graziano A, D'Aquino R, Laino G and Papaccio G: Dental pulp stem cells: A promising tool for bone regeneration. Stem Cell Rev 4: 21-26, 2008.

31. Morad G, Kheiri L and Khojasteh A: Dental pulp stem cells for in vivo bone regeneration: A systematic review of literature. Arch Oral Biol 58: 1818-1827, 2013.

32. Sculean A,Chiantella GC,Windisch P, Gera I and ReichE: Clinical evaluation of an enamel matrix protein derivative (Emdogain) combined with a bovine-derived xenograft (Bio-Oss) for the treatment of intrabony periodontal defects in humans. Int J Periodontics Restorative Dent 22: 259-267, 2002.

33. Valentini P and Abensur DJ: Maxillary sinus grafting with anorganic bovine bone: A clinical report of long-term results. Int J Oral Maxillofac Implants 18: 556-560, 2003.

34. Klinge B, Alberius P, Isaksson S and Jönsson J: Osseous reponse to implant natural bone mineral and synthetic hydroxylapatite ceramic in the repair of experimental skull bone defects. J Oral Maxillofac Surg 50: 241-249, 1992.

35. Jensen SS, Aaboe M, Pinholt EM, Hjørting-Hansen E, Melsen F and Ruyter IE: Tissue reaction and material characteristics of four bone substitutes. Int J Oral Maxillofac Implants 11: 55-66, 1996.

36. Berglundh $\mathrm{T}$ and Lindhe $\mathrm{J}$ : Healing around implants placed in bone defects treated with Bio-Oss. An experimental study in the dog. Clin Oral Implants Res 8: 117-124, 1997.

37. Piattelli M, Favero GA, Scarano A, Orsini G and Piattelli A: Bone reactions to anorganic bovine bone (Bio-Oss) used in sinus augmentation procedures: A histologic long-term report of 20 cases in humans. Int J Oral Maxillofac Implants 14: 835-840, 1999.

38. Cowan CM, Shi YY, Aalami OO, Chou YF, Mari C, Thomas R, Quarto N, Contag CH, Wu B and Longaker MT: Adipose-derived adult stromal cells heal critical-size mouse calvarial defects. Nat Biotechnol 22: 560-567, 2004.

This work is licensed under a Creative Commons Attribution-NonCommercial-NoDerivatives 4.0 International (CC BY-NC-ND 4.0) License. 